\title{
炔烃与二醇通过碳-碳参键裂解的电化学酯化反应
}

\author{
唐海涛潘英明* \\ (广西师范大学化学与药学学院 广西桂林 541004)
}

\section{Electrochemical Esterification Reaction of Alkynes with Diols via Cleavage of Carbon-Carbon Triple Bonds}

\author{
Tang, Haitao Pan, Yingming*
}

(School of Chemistry and Pharmaceutical Sciences, Guangxi Normal University, Guilin, Guangxi 541004)

碳一碳键的裂解反应作为一种有用的合成工具可用 于构建许多有价值的化合物, 该类反应目前受到了许多 关注, 并在农药、药物和材料领域得到了广泛应用 ${ }^{[1]}$. 在 过去的几十年中, 碳一碳单键和碳一碳双键的裂解反应已 经得到了深入的研究与发展 ${ }^{[1-2]}$. 然而, 由于碳-碳参键 的键能很大, 其裂解反应发展较缓慢, 目前仍然是有机 化学领域中最具挑战性的课题之一. 炔烃的复分解反应 是碳-碳参键裂解反应的例子之一 ${ }^{[3]}$, 但是, 该类反应需 要使用过渡金属催化剂, 例如, 通常使用钼或铇等. 除 复分解反应外, 碳一碳参键裂解反应的例子很少. 例如, 2001 年韩国延世大学 Jun 课题组 ${ }^{[4]}$ 报道了通过铑催化的 碳一碳参键裂解的加氢亚氨基酰化反应. 从这些已报道 的文献可以看出, 过渡金属催化在碳一碳参键裂解反应 中起着一个重要的作用. 然而, 这些昂贵或有毒的过渡 金属的使用增加了产物成本和纯化难度. 另外, 这些反 应大多数需要苛刻的反应条件. 目前已经报道了一些避 免使用过渡金属催化剂的碳-碳参键裂解反应的例子 ${ }^{[5]}$, 但是, 许多反应仍然需要大量的氧化剂. 由于现有的碳 一碳参键裂解反应大多数涉及过渡金属催化剂和大量氧 化剂的使用, 因此开发绿色和可持续的碳一碳参键裂解 反应很有必要.

关于炔烃通过碳一碳参键断裂的酯化反应, 目前仅 报道了两种方法. 2008 年, 华南理工大学化学与化工学 院江焕峰课题组 ${ }^{[6]}$ 开发了一种钯催化的碳-碳参键与氧 分子的裂解反应(Scheme 1a), 但是, 该反应存在许多缺 点: (1)反应使用了昂贵的过渡金属催化剂 $\operatorname{Pd}(\mathrm{OAc})_{2} ;(2)$ 反应条件苛刻, 需要高压和高温; (3)反应需要高压氧气 作为氧化剂和 $\mathrm{ZnCl}_{2} \cdot 2 \mathrm{H}_{2} \mathrm{O}$ 作为添加剂. 除了以上缺点
外，该方法没有使用二醇作为底物参与反应. 2014 年, 湖南大学化学化工学院郭灿城课题组 ${ }^{[7]}$ 报道了 [双 (三氟 乙酰氧基)碘]苯(PIFA)介导的通过碳一碳参键氧化裂解 的炔烃与醇的酯化反应(Scheme 1b), 但是, 该反应使用 了大量的氧化剂(3.5 equiv. PIFA), 并且没有使用二醇作 为反应底物。

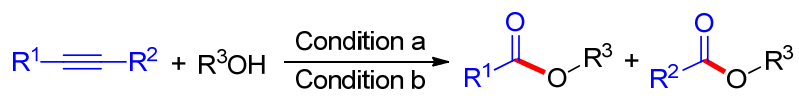

Condition a: $\mathrm{Pd}(\mathrm{OAc})_{2}, \mathrm{O}_{2}(758 \mathrm{kPa}), \mathrm{ZnCl}_{2} \cdot 2 \mathrm{H}_{2} \mathrm{O}, 100^{\circ} \mathrm{C}, 24 \mathrm{~h}$ Condition b: $\mathrm{Phl}\left(\mathrm{OCOCF}_{3}\right)_{2}\left(3.5\right.$ equiv.), $60^{\circ} \mathrm{C}, 15 \mathrm{~h}$

图式 1 炔烃通过碳一碳参键断裂的酯化反应的传统方法 Scheme 1 Traditional method of esterification reaction of alkynes via cleavage of $\mathrm{C}-\mathrm{C}$ triple bonds

近年来, 电化学合成因其绿色环保和经济的特性已 经发展成为一种重要的合成方法. 电化学提供了一种直 接在电极上转移电子的经济方法. 因此, 可以通过阳极 氧化直接进行许多反应, 而无需使用催化剂、氧化还原 介质或氧化剂. 2018 年, 厦门大学化学化工学院徐海超 课题组 ${ }^{[8]}$ 实现了烯烃与二醇的电化学偶联反应(Scheme 2a), 然而, 炔烃和二醇的电化学反应从未报道过, 并且 徐海超课题组报道的反应得到的是环状产物而不是酯. 其中, 如何避免形成环状产物具有一定的挑战性.

鉴于以上报道及其存在的问题, 最近, 淮北师范大 学化学与材料科学学院李品华课题组 ${ }^{[9]}$ 报道了在不使用 催化剂、氧化剂和添加剂的情况下, 二芳基乙炔与二醇 及其衍生物发生的新型电化学酯化反应(Scheme 2b).

该方法具有无催化剂、无氧化剂、无添加剂和原子

* Corresponding author. E-mail: panym@mailbox.gxnu.edu.cn. Published online January 5, 2021 
(a) Electrochemical intermolecular annulation reaction of alkenes with diols

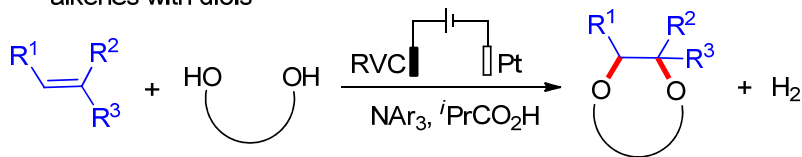

(b) Electrochemical esterification reaction of alkynes with diols via cleavage of $\mathrm{C}-\mathrm{C}$ triple bonds

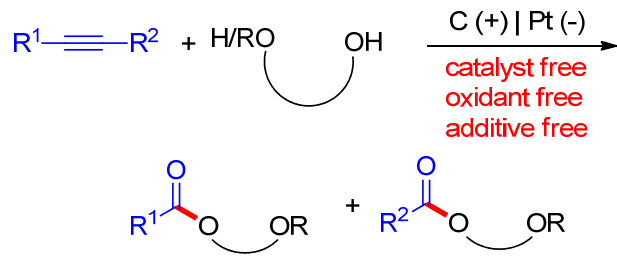

图式 2 二醇与烯烃或炔烃的电化学反应

Scheme 2 Electrochemical reaction of diols with alkenes or alkynes

经济等特点，因此是绿色环保的. 该反应是通过碳一碳 参键断裂的电化学反应的第一个例子，也是首次实现了 炔烃与二醇的碳一碳参键裂解反应, 并且, 该反应构建 了一系列的酯类化合物，产率高达 $80 \%$ 。

机理研究如 Scheme 3 所示：二芳基乙炔 $\mathbf{1}$ 通过阳极 氧化转化为自由基阳离子 $\mathbf{A}$, 然后自由基阳离子 $\mathbf{A}$ 与试 剂中的水发生脱质子化作用生成中间体 B. 随后中间体 $\mathbf{B}$ 进一步阳极氧化形成中间体 $\mathbf{C}$. 在阴极, 乙二醇 2 转 化为阴离子 $\mathbf{D}$ 和 $\mathrm{H}_{2}$, 然后 $\mathbf{D}$ 经过阳极氧化形成自由基 $\mathbf{E}$. 最后，自由基 $\mathbf{E}$ 与中间体 $\mathbf{C}$ 反应生成酯类产物 $\mathbf{3}$.

李品华课题组的这一研究成果为炔烃的碳一碳参键 裂解反应开发了一条更加绿色经济的合成途径, 并且拓 宽了碳-碳参键与醇类底物的反应类型.

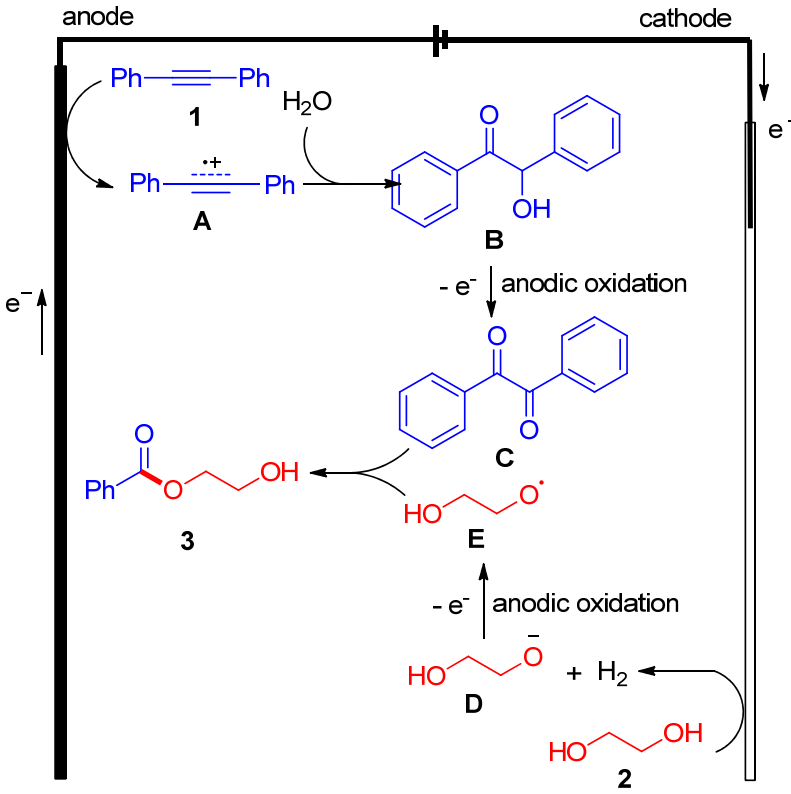

图式 3 反应的可能机理

Scheme 3 Plausible reaction mechanism

\section{References}

[1] Souillart, L.; Cramer, N. Chem. Rev. 2015, 115, 9410.

[2] Yu, Q.; Zhang, Y.; Wan, J. P. Green Chem. 2019, 21, 3436.

[3] Fürstner, A. Angew. Chem., Int. Ed. 2013, 52, 2794.

[4] Jun, C. H.; Lee, H.; Moon, C. W.; Hong, H. S. J. Am. Chem. Soc. 2001, 123, 8600.

[5] Katta, N.; Ojha, M.; Murugan, A.; Arepally, S.; Sharada, D. S. RSC Adv. 2020, 10, 12599

[6] Wang, A.; Jiang, H. J. Am. Chem. Soc. 2008, 130, 5030.

[7] Jiang, Q.; Zhao, A.; Xu, B.; Jia, J.; Liu, X.; Guo, C. J. Org. Chem. 2014, 79, 2709.

[8] Cai, C. Y.; Xu, H. C. Nat. Commun. 2018, 9, 3551.

[9] Wang, P. L.; Shen, H. Z.; Cheng, H. H.; Gao, H.; Li, P. H. Green Chem. 2020, 22, 6783. 